
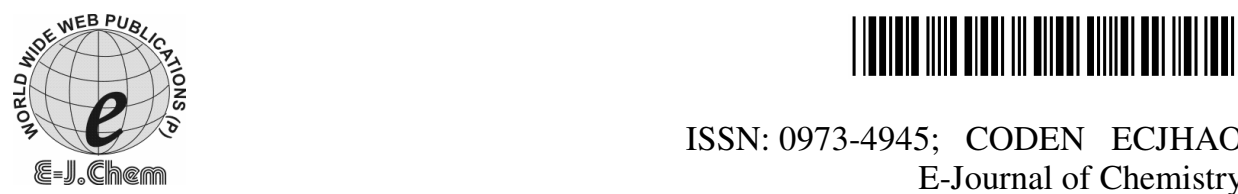

ISSN: 0973-4945; CODEN ECJHAO

http://www.e-journals.net

E-Journal of Chemistry

2009, 6(1), 169-176

\title{
Linnett Double Quartet Theory, Challenging the Pairing Electrons
}

\author{
YEGANEH KHANIANI and ALIREZA BADIEI* \\ School of Chemistry, University College of Science, \\ University of Tehran,Tehran, Iran \\ abadiei@khayam.ut.ac.ir
}

Received 28 May 2008; Accepted 20 July 2008

\begin{abstract}
Linnett proposed his theory 45 years after Lewis's supposition. During these years Pauling, Mulliken and Hund made different molecular structure models based on valance bond and molecular orbital theory. Their theories were seemed to fit many experiences but had some inabilities. Linnett's double quartet theory could solved some important problems about stability of radicals, paramagnetism / diamagnetism in molecular systems, electronic structure in transition states and finally challenge of resonance and aromaticity. In this article we review some basic ideas and concepts concerning different models and then we give a detailed discussion of the LDQ and use it in diverse branches of chemistry.
\end{abstract}

Keyword: Linnett's double quartet theory, LDQ, Valence bond theory and Aromatisity.

\section{Introduction}

In 1916 Lewis proposed his formula based on the "group of eight" which was contained electron pairs; sometimes shared, sometimes lone. Lewis stressed the importance of "the pairing of electrons". However, he was unable to explain the stability of molecules or radicals such as nitric oxide ${ }^{1}$. In 1931 Pauling presented the valence bond theory (VB) that was closely concerned to the bond concept. When resonance was proposed and accepted as an important part of Valence bond theory, the concept of the electron-pair was retained. All the contributing structures were made up of electron pairs ${ }^{2}$. At the same time, Mulliken and Hund built their modern molecular orbital (MO) theory. The VB theory gives a simple and very approximate description of the bonding in a molecule, but in general it does not predict geometry. For molecules such as $\mathrm{CH}_{4}, \mathrm{NH}_{3}$ and $\mathrm{H}_{2} \mathrm{O}$, this assumption does not predict the correct shapes. The multiple bonds in ethane and ethylene are, however, described using a type of hybrid MO-VB description. This description introduces the overlapping of the orbital concepts in a sideway manner and the concept of hybridization of orbitals. Therefore, both the VB and MO theories have the limitations for understanding molecular geometry. Thus was introduced another model, Valence-Sell Electron-Pair Repulsion (VSEPR). 


\section{The electron repulsion theory}

The Pauli principle determines the most probable distribution of the electrons in a valence shell. In a $\mathrm{AX}_{\mathrm{n}}$ molecule in which $\mathrm{A}$ has an octet of electrons and there are at least two ligands $\mathrm{X}$, the Pauli principle together with electrostatic attraction between the electrons and the positive core of the ligands, leads to the formation of typical configuration that is shown ${ }^{3}$ in Figure 1.

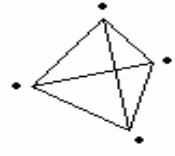

a

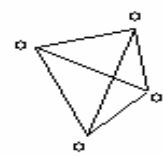

$\mathrm{b}$

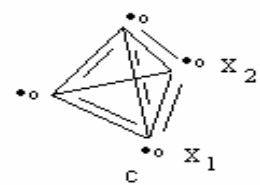

c:

Figure 1. a) Four $\alpha$-spin electrons, b) Four $\beta$-spin electrons, c) In the presence of nuclei of two ligands such as $\mathrm{H}_{2} \mathrm{O}$, two tetrahedral are adapted at all four tetrahedral positions.

In 1961 J.W. Linnett presented his double quartet theory as a simple modification of Lewis's original bonding concept. He demonstrated that the concept of hybrid atomic orbital is unnecessary and provided that the habit of pairing electrons whenever possible be neglected. When arrangement of electrons looks like the cube, two electrons of opposite spin, having less repulsion than two electrons of the same spin. If have another view of Figure 2, it will be two tetrahedron. And in a different view we would have two interpenetrating trigonal pyramids so $\mathrm{s}$ and $\mathrm{p}$ electrons are at the apexes and in the bases of the pyramids respectively.
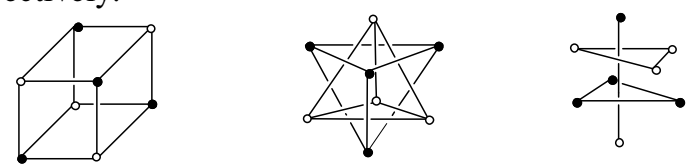

Figure 2. Three different view of Linnett electron arrangement.

\section{Atomic model for transition metals}

Linnett model for another elements of periodic table is interesting too. The icosahedron is the pattern for d block elements, Sc to $\mathrm{Zn}$. Ten d electrons of $\mathrm{Zn}$ atom are on the pentagonal bases and two s electrons are at the apexes (Figure 3a). For the arrangement of the f block elements, see ${ }^{4}$ Figure $3 b$.

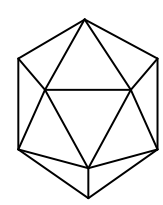

a
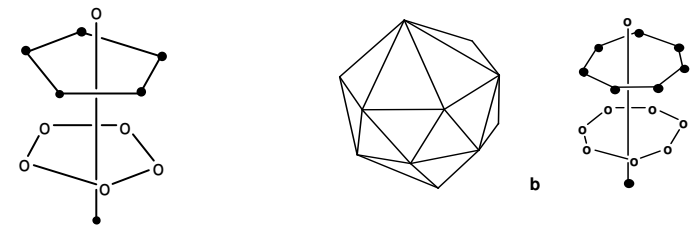

Figure 3. Atomic model for (a) d block and (b) f block elements.

\section{The principles of $L D Q$ theory}

a) The octet electrons of valance shell for each atom should be considered double quartet set. One set having $\alpha$ spin and another $\beta$ spin.

b) Each set of four individually have a tetrahedral arrangement because the electrostatic repulsion and spin correlation cancel one another.

c) Electrons of one spin set which are participating in a double bond are located at the vertices of two tetrahedral so have an edge in common between the two nuclei. Similarly those participating in a triple bond may be arranged on two tetrahedral distributions having a face in common between the two nuclei. 

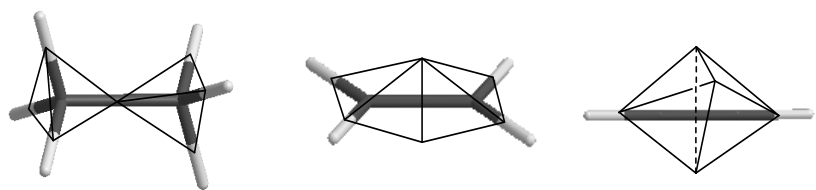

Figure 4. Single, double and triple bond in ethane, ethylene and acetylene respectively left to right.

d) Because of repulsion between electrons, if the two tetrahedral spin sets round a nucleus assume different dispositions, the energy will be lower than if they are close to one another.

e) The two spin sets of electrons will tend to adopt a regular tetrahedral configuration, and any deviation from this will involve an increase of energy.

f) The formal charge of an atom in a molecule is calculated by supposing that each lone electron contributes its full charge to the atom with which it is associated, and each shared electron contributes half its charge to each of the bonded atoms ${ }^{1}$.

\section{Kinds of bond in $L D Q$}

The chemical bond between atoms is defined in terms of the electron-electron, nuclearnuclear, and electron-nuclear electrostatic forces. The electron-nuclear force is just the force of attraction between atoms, but there are two intra-particle forces of repulsion ${ }^{5}$. The nuclei in this theory, play an important role that should not be neglected. On the one hand, electrons have a tendency to anticoincidence and reducing the repulsion. On the other hand, they trend to increase the electron attraction and decrease the nuclei-nuclei repulsion by concerning the electrons during intra-nucleus axes. The electron correlation is determined by tendency of electrons and nucleuses to the most number of electrons between atoms. Thus electrons of the opposite spin may be or not to be closed together and consequently more electrons can be associated between two atoms to form a bond.

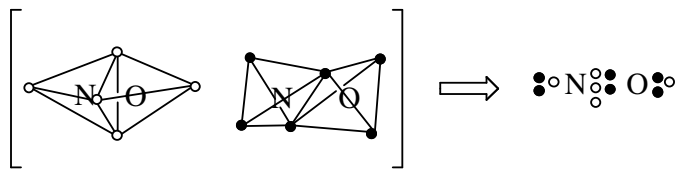

Figure 5. Linnett's performance of nitrogen oxide.

The practical consequences of this are the appearance of valence electrons which are not close paired and the possibility of one, three, and five electron bonds in addition to the close-paired two, four, and six electron bonds. Linnett's formula for NO gives a simple explanation of a molecule which has one more electron of one spin than of the other, and therefore it is paramagnetic. His structures (Figure 5) are consistent with the bond order that is average of double and triple bond ${ }^{4}$

\section{Application of $L D Q$ in organic chemistry}

Double quartet theory could predict easily paramagnetism/diamagnetism, molecular structures and radical species stability by simultaneous conjugation with electron donor and acceptor groups. However, the power of this theory is not be limited to inorganic chemistry. It is more impressive Linnett's solution of the ancient riddle of the benzene molecule without resonance or $\pi$ electrons. Meanwhile we can analysis the transition states of organic reactions like $S_{N} 2$ by introducing simple concepts such as $L$-strain. In this review, we evaluate these different aspects of LDQ theory, briefly. 


\section{L-Strain}

Any event at a given point in the valence shell of an atom, which alters the relative disposition of two spin sets at that point will alter it at all other points as well, in a definite way. Thus all the bonds in the atom play a role in the reaction, but this factor dose not replace the usual steric and electronic effects. Sometimes two electrons in a single bond are forced separate and localized out of internuclear line. This bond weakening, raise the activation energy and is called $L$-strain.

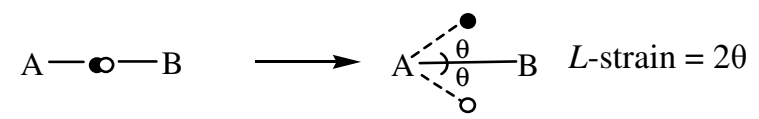

Many of molecules such as cyclopropane, cyclobutane, ethylene and acetylene suffer from $L$-strain at each carbon atom. The Figure 6 presents the calculation of this parameter for each case. A correction must be made for electron correlation was discussed earlier. For a first-row atom, ideal correlation is achieved at $2 \theta=70.5^{\circ}$, due to the cubical arrangement of the valance shell electrons, any further increase in $\theta$ unavoidably involves the nearer approach of other electrons of the valance shell. It can be obtained $4 \mathrm{kcal} / \mathrm{mol}$ per electron pair, from the resonance energy of benzene which is assigned to $2 \theta=70.5^{\circ}$. The angle strain in cyclopropane has been predicted to be about $21 \mathrm{kcal} / \mathrm{mol}$, which means $7 \mathrm{kcal} / \mathrm{mol}$ per $\mathrm{C}-\mathrm{C}$ bond. $2 \theta$ in cyclopropane must be less than $49.5^{\circ}$, the correlation correction according this angle then, is about $3 \mathrm{kcal} / \mathrm{mol}$ per bond and the corrected $L$-strain energy for cyclopropane is $4 \mathrm{kcal} / \mathrm{mol}$ per electron pair. The angle strain energy of cyclobutane is almost the same as that of cyclopropane. Thus the $L$-strain energy for $2 \theta=109.5-90=19.5^{\circ}$ comes around $4 \mathrm{kcal} / \mathrm{mol}$ per electron pair.
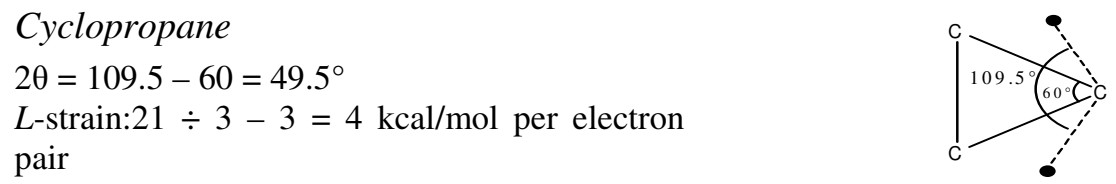

\section{Cyclobutane}

$2 \theta=109.5-90=19.5^{\circ}$

$L$-strain : $21 \div 4-1.2=4 \mathrm{kcal} / \mathrm{mol}$ per electron pair

\section{Ethylene}

$2 \theta=109.5^{\circ}$

$L$-strain: $19.4 \div 2=9.7 \mathrm{kcal} / \mathrm{mol}$ per electron pair

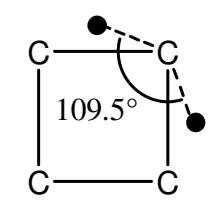

\section{Acetylene}

$2 \theta=109.5^{\circ}$

$L$-strain : $48.2 \div 3=16.1 \mathrm{kcal} / \mathrm{mol}$ per electron pair
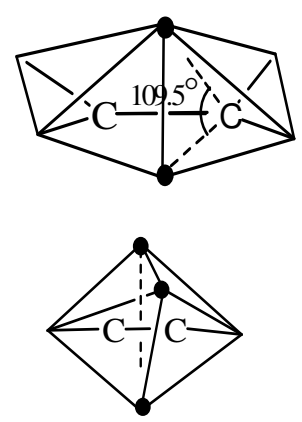

Figure 6. Presentation and calculation of $L$-strain for some organic molecules. 
And about ethylene, the difference in bond energy between one double bond and two single $\mathrm{C}-\mathrm{C}$ bonds is $19.4 \mathrm{kcal} / \mathrm{mol}$. ethylene as was shown in figure 4 has $2 \theta=109.5^{\circ}$, there is zero correlation correction for this angle. The $L$-strain energy comes to $9.7 \mathrm{kcal} / \mathrm{mol}$ per electron pair. Acetylene has six correlated electrons in the triple $\mathrm{C}-\mathrm{C}$ bond, each with $\theta$ $\approx 70.5^{\circ}$ ( 3 electron in another spin was not shown). The difference in bond energy between three single bonds and one triple bond is $48.2 \mathrm{kcal} / \mathrm{mol}$. Without the correlation, the $L$-strain is $16.1 \mathrm{kcal} / \mathrm{mol}$ per electron pair ${ }^{6}$.

\section{Transition state}

Another feature of Linnett's theory is prediction of products configuration in reactions such as Elimination and nucleofilic substitution. One of the most common devices in this prediction is the transition state picture. For E2 reaction, two planar transition states are possible (Figure 7). The trans transition state has higher electron correlation, though the cis one is strained, because of electron distortion from staggered tetrahedral arrangement ${ }^{7}$.

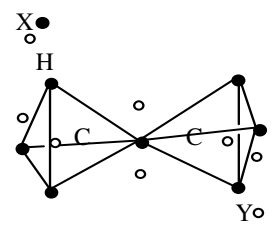

trans

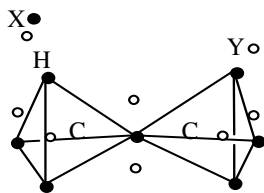

cis

Figure 7. Cis and trans transition state electronic structures in Elimination.

As you know, the $S_{N} 2$ reaction take place by two possibility, retention and or inversion of configuration. We should look to the transition state again. The transition state in inversion route in classic view and in new presentation is shown in Figure 8. The classic structure has two problems which are often ignored. One is the number of electrons in the five bonds that is ten. The other is activation energy that is necessary in formation of the new bond and dissolution of the old bond coincidently.<smiles>[Y]C([Y])([2H])[Pb]</smiles><smiles>[Y]C([2H])(P)C([Y])(P)P</smiles>

Figure 8. Classic presentation (left) and new suggested structure (right) of transition state in $\mathrm{S}_{\mathrm{N}} 2$ reaction.

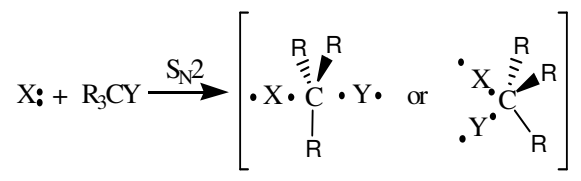

Although the number of bonding electrons is constant during the transformation, but activation energy is required in this exothermic direction because a one-electron bond is not necessarily one-half as strong as a two-electron bond. For anticipation of mechanism we should make estimation of difference in $L$-strain in the transition state for inversion and retention. In the double quartet representation of the transition state for a inversion mechanism, each $\mathrm{C}-\mathrm{R}$ bond suffers from $40^{\circ}$ of $L$-strain that is correspond to $3.6 \mathrm{kcal} / \mathrm{mol}$ for a $\mathrm{C}-\mathrm{C}$ bond and $4.3 \mathrm{kcal} / \mathrm{mol}$ for a $\mathrm{C}-\mathrm{H}$ bond. Thus for $\mathrm{S}_{\mathrm{N}} 2$ displacement on methyl about $13 \mathrm{kcal} / \mathrm{mol}$ of $L$-strain in the tree $\mathrm{C}-\mathrm{H}$ bonds is predicted. A correlation correction 
has been made for the $L$-strained bonds. The fourth electron pair around carbon is well correlated in the transition state but was frankly paired in the starting molecule. It must be deduced $4 \mathrm{kcal} / \mathrm{mol}$ for this correction. Then the net energy is about $9 \mathrm{kcal} / \mathrm{mol}$ when $\mathrm{R}=\mathrm{H}$. For a retention mechanism, $L$-strain would be much higher, $c a .70 .5^{\circ}$. This strain should constitute the major portion of the activation energy in the absence of external factors such as solvation. Thus the activation energy for retention will be greater than that for inversion by $c a .11 \mathrm{kcal} / \mathrm{mol}$ for $\mathrm{R}=\mathrm{H}$. Therefore inversion is almost preferred, although retention is not forbidden and should be considered under the proper conditions ${ }^{6-8}$.
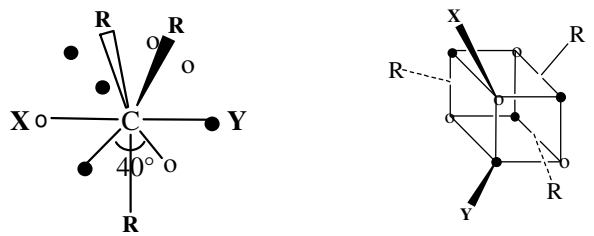

Figure 9. LDQ presentation of transition state for inversion (left) and retention of configuration (right).

\section{Radicals}

The stability of organic free radicals that is unexplained with valance shell theory has explained with Linnett's three or five-electron bonds. O, $\mathrm{N}$ and halogens, even carbon will stabilize a radical when it possesses unshared electrons. The geometry of trivalent carbon in methyl radical with a septet of electrons can be predicted in simple terms from the Linnett structure. One spin set is a triangle and the other a tetrahedron, all three bonds are L strained by $19.5^{\circ}$. The molecule will not be flattened pyramid and easily inverted by rotating one spin set as was shown ${ }^{6,7}$ in Figure 10.

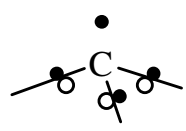

Figure 10. Arrangement of electrons in methyl radical.

\section{Riddle of benzene}

The Lewis structure for the benzene molecule is in which the $\mathrm{C}-\mathrm{C}$ bonds are alternately single and double and should therefore have lengths of $154 \mathrm{pm}$ and $134 \mathrm{pm}$, respectively. But the $\mathrm{C}-\mathrm{C}$ bonds are found experimentally to all have the same length of $140 \mathrm{pm}$, which is intermediate between the $\mathrm{C}-\mathrm{C}$ single and double bond lengths. The reason for these equal bond lengths is that electrons are not always as localized as the Lewis structure implies. Thus we can write two different but equivalent Lewis structure for benzene.<smiles>Cc1c(C)c(C)c(C)c(C)c1C</smiles>

A<smiles>Cc1c(C)c(C)c(Br)c(C)c1C</smiles>

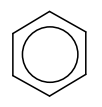

C

According to these two structures a given $\mathrm{C}-\mathrm{C}$ bond may be considered to be either a single bond or a double bond. This implies that there are three electron pairs that can not be considered to be localized between three particular pairs of carbon atoms but must be considered to be delocalized over all six bonding regions. This two structures are called resonance structures. On the other hand, according to the basic principles of resonance theory, the position of nuclei must be the same in all the structures while the electron localization in $\mathrm{A}$ and $\mathrm{B}$ decreases the symmetry from D6h to D3h, and symmetry $\mathrm{C}$ 
Woodward-Haffmann rules to dynamic resonance between the two Kekule formula.These rule $\mathrm{s}$ is related to the problem of aromaticity and antiaromatisity in resonance theory. The generalized selection rule in Woodward-Haffmann: "A ground state pericyclic change is symmetry-allowed when the total number of $(4 q+2)_{s}$ and $(4 r)_{a}$ components is odd. In other words, only the number of electrons involved in a reaction and the stereochemistry of the initial and final products are taken into account, without any mention of symmetry. In a purely classical language "pericyclic reactions are allowed when the sum of the number of electron pairs involved and of odd processes is odd. Odd processes such as antrafacial, conrotatory processes that are involving an odd number inversions of configuration. Figure 8 shows the dynamic resonance between the two limiting structures. Since there are three electron paires in this reaction, we have to look for a new model that could definite such odd processes. This model including two Walden inversion and can be used as WH-allowed pericyclic reaction.

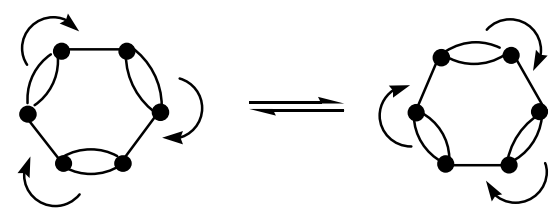

Figure 11. Dynamic resonance between two bent-bond Kekule formulae.

And about Linnett's double quartet picture of benzene that uses a localized representation of the electrons. $\sigma / \pi$ separation cannot be introduced in a localized picture, and must be concluded that the double-quartet model should have been applied to the total system of electrons. In this new formula of benzene we have "non-paired spatial orbitals" instead of $\sigma / \pi$ system (Figure 12$)^{1,9}$.

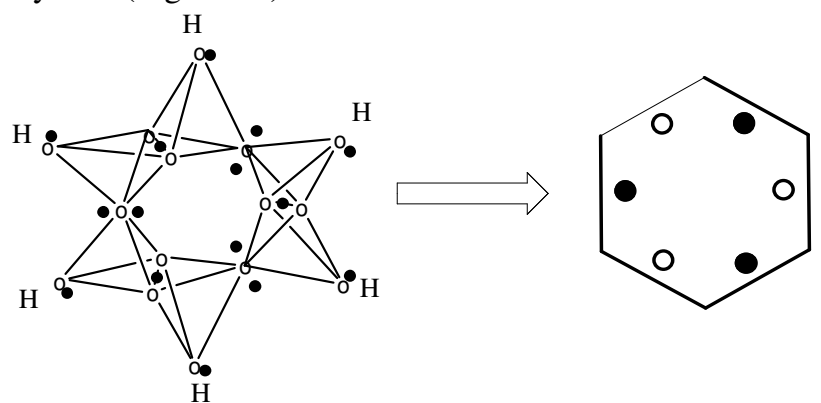

Figure 12. Linnett's representation of benzene molecule with three-electron bonds.

\section{Conclusion}

Linnett has explained the structure of molecules in a simple way. The arrangement of electrons in valence shell is analogous for $s, \mathrm{p}$ and $\mathrm{f}$ block elements. The power of LDQ theory is in describing the electronic structures of molecules, especially those with $\pi$ systems. The Linnett theory is compatible with the valence bond and molecular orbital viewpoints. Deciding about which models, quantum or classical can describe our molecule and reaction is an easy and important choice. Double quartet theory could predict easily paramagnetism/diamagnetism, molecular structures and radical species stability by simultaneous conjugation with electron donor and acceptor groups. However, the power of this theory is not be limited to inorganic chemistry. It is more impressive Linnett's solution of the ancient challenge of the benzene molecule without resonance or $\pi$ electrons. Meanwhile we can analysis the transition states of organic reactions like $S_{N} 2$. 


\section{References}

1 Linnett J W, J Am Chem Soc., 1961, 83, 2643.

2 Gillespie R G, Hargittai I, The VSEPR Model of Molecular Geometry; 1991 by Allyn \& Bacon, Needham Heights, Massachusetts 02194.

3 Gillespie R J, J Chem Educ., 2004, 81, 3, 289.

4 Luder W F, J Chem Educ.,.1967, 44, 206.

5 Bader R F W, Hernandez-Trujillo J and Cortes-Guzman F, J Comput Chem., 2006, 28, 1,4 .

$6 \quad$ Firestone R A, J Org Chem., 1969, 34, 9, 2621.

7 Firestone R A, Tetrahedron Lett., 1968, 8, 971.

8 Fireston R A, J Org Chem., 1971, 36, 5, 702.

9 Rassat A, Phys Chem Chem Phys., 2004, 6, 232. 


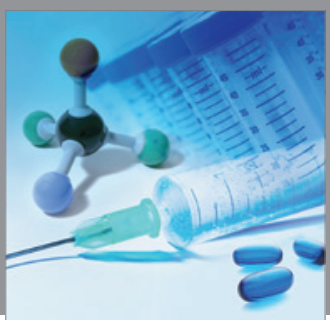

International Journal of

Medicinal Chemistry

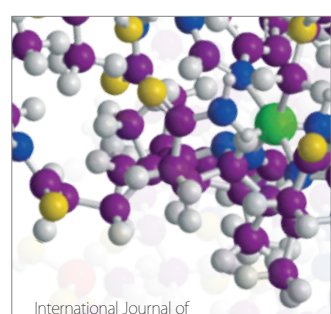

Carbohydrate Chemistry

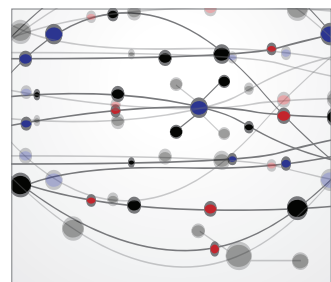

The Scientific World Journal
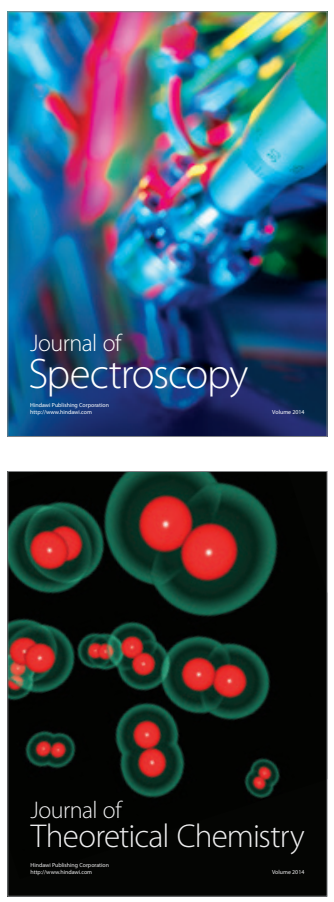
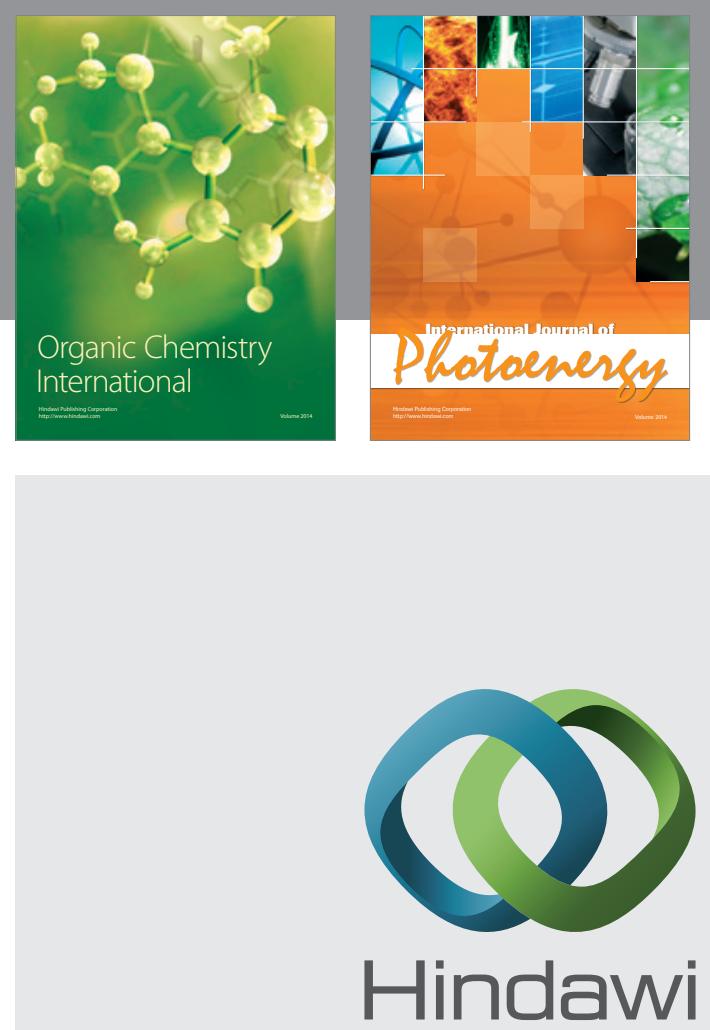

Submit your manuscripts at

http://www.hindawi.com
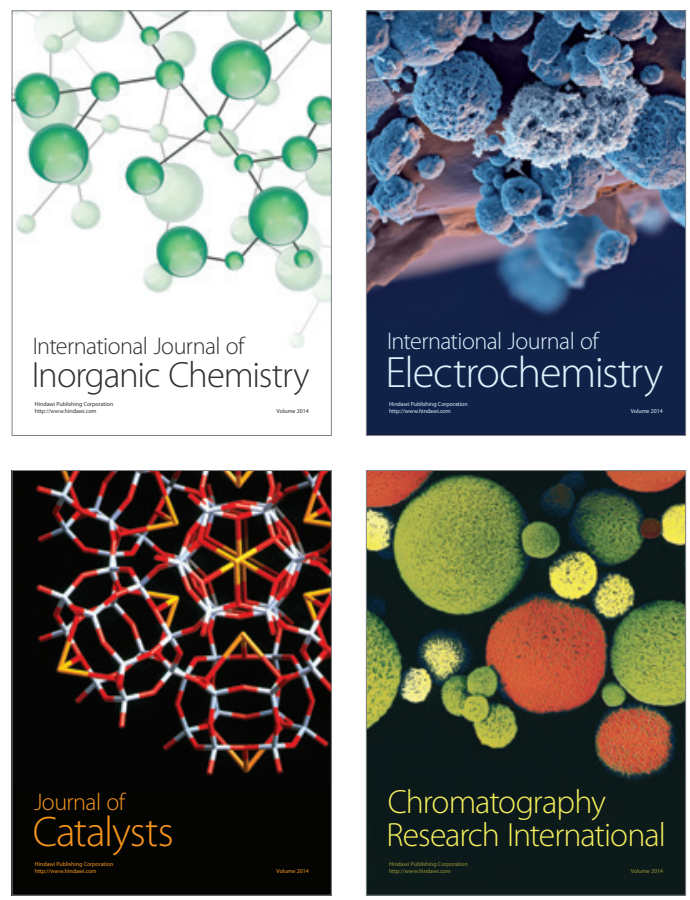
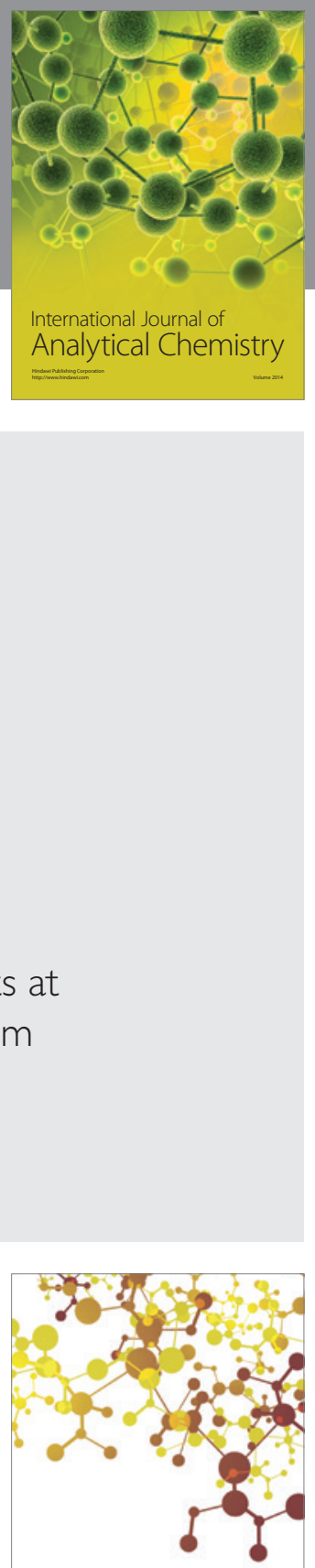

Journal of

Applied Chemistry
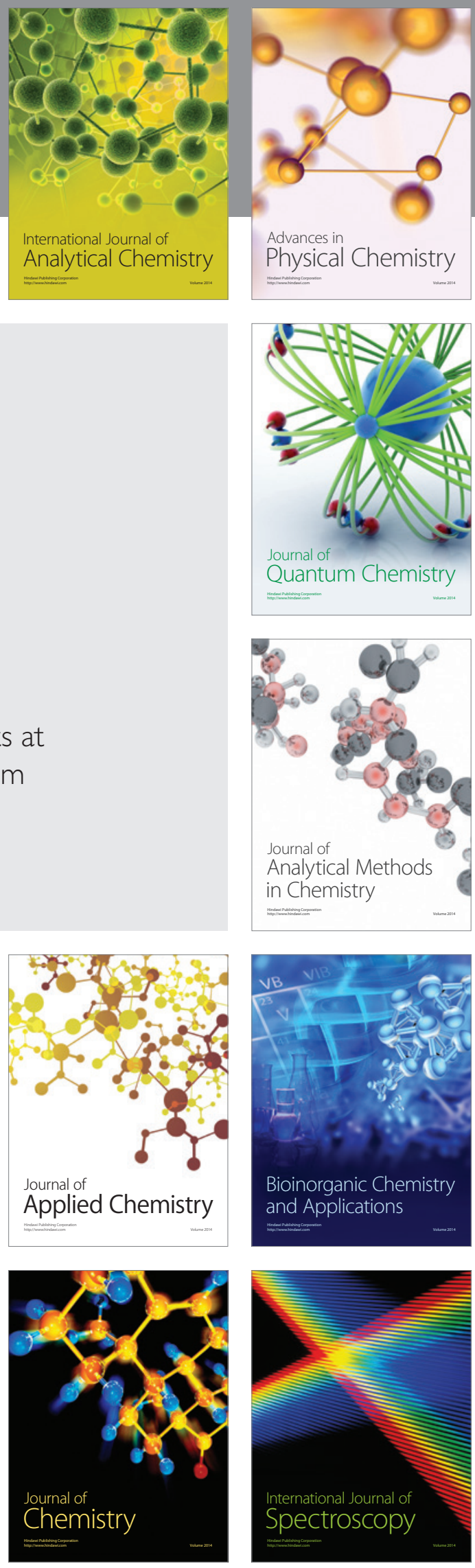